\title{
DEFENSE TECHNICAL INFORMATION CENTER
}

Informution for the Defense Community

$D_{\text {DTIC }}{ }^{\circledR}$ has determined on $03 / 3 / 12009$ that this Technical Document has the Distribution Statement checked below. The current distribution for this document can be found in the DTIC ${ }^{\circledR}$ Technical Report Database.

Distribution Statement A. Approved for public release; distribution is unlimited.

(c) COPYRIGHTED; U.S. Government or Federal Rights License. All other rights and uses except those permitted by copyright law are reserved by the copyright owner.

DISTRIBUTION STATEMENT B. Distribution authorized to U.S. Government agencies only (fill in reason) (date of determination). Other requests for this document shall be referred to (insert controlling DoD office)

DISTRIBUTION STATEMENT C. Distribution authorized to U.S. Government Agencies and their contractors (fill in reason) (date of determination). Other requests for this document shall be referred to (insert controlling DoD office)

DISTRIBUTION STATEMENT D. Distribution authorized to the Department of Defense and U.S. DoD contractors only (fill in reason) (date of determination). Other requests shall be referred to (insert controlling DoD office).

DISTRIBUTION STATEMENT E. Distribution authorized to DoD Components only (fill in reason) (date of determination). Other requests shall be referred to (insert controlling DoD office).

DISTRIBUTION STATEMENT F. Further dissemination only as directed by (inserting controlling DoD office) (date of determination) or higher DoD authority.

Distribution Statement $F$ is also used when a document does not contain a distribution statement and no distribution statement can be determined.

DISTRIBUTION STATEMENT X. Distribution authorized to U.S. Government Agencies and private individuals or enterprises eligible to obtain export-controlled technical data in accordance with DoDD 5230.25; (date of determination). DoD Controlling Office is (insert controlling DoD office). 


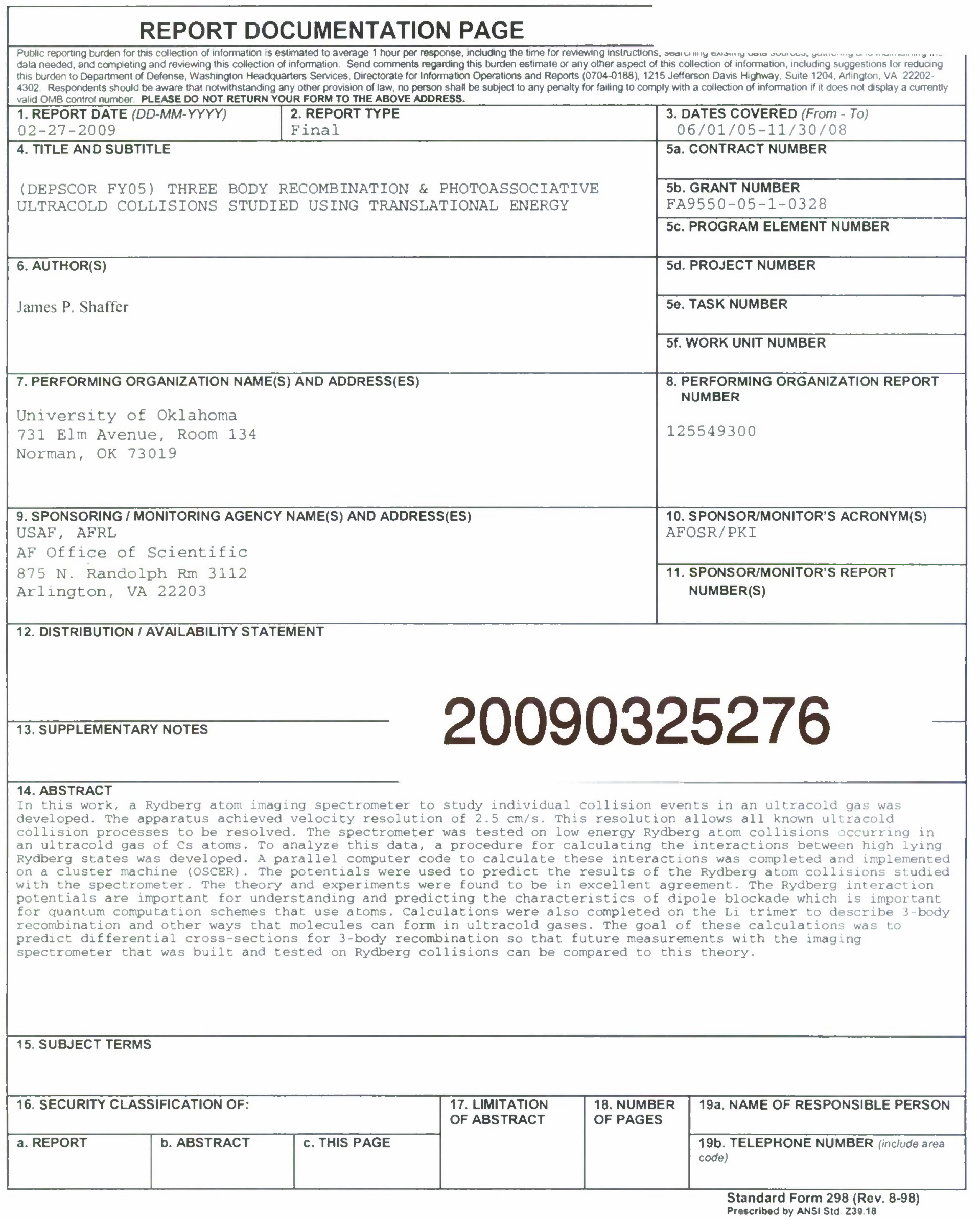




\section{$\underline{\text { Report }}$}

We have constructed our cold atom imaging spectrometer, realizing less than $2.5 \mathrm{~cm} / \mathrm{s}$ velocity resolution [1]. This result is an order of magnitude higher resolution than obtained anywhere prior to our work. The high resolution enables the study of ultracold collisions using Rydberg atom imaging. We have used our machine to study elastic and inelastic collisions in cold Rydberg gases [2]. These measurements were compared to calculations of Rydberg atom pair interactions that included a background electric field. We were the first group to calculate non-perturbative interaction potentials between Rydberg atoms with high principle quantum number in a background clectric field [3]. An understanding of cold Rydberg atom interactions is important for using these samples for quantum computation. In the course of this work, we discovered that long range $(\sim 5-10 \mu \mathrm{m}$ internuclear separation) Rydberg atom molecules could be formed by applying a background electric field to shift the Rydberg atom energy levels [4]. This important finding was verified using a variation of Coulomb explosion imaging with the apparatus [5]. Without a differential technique it is experimentally difficult to distinguish a collision resonance from a molecular resonance in cold Rydberg atom spectra because the vibrational energy spacing of the molecules is very small. Our apparatus was the first to achieve this objective.

We are currently extending our work on Rydberg atom imaging to the study ultracold three-body recombination. Rydberg atom collisions are a good test of our methods because they exhibit cxtremely low encrgy collisions. Three-body recombination in cold alkali gases is among the most important loss mechanisms in a BEC. These processes are consequently important for using Bose-Einstein condensates as sensors and for fundamental experiments that require large numbers of atoms. There are currently no measurcments of state-tostate differential cross-sections for 3-body recombination in an alkali gas at ultracold temperatures. In this grant cycle, we calculated potentials for triatomic alkali systems [6,7] and investigated ways to coherently control the formation of molecules in the cold gas [8,9]. As the calculations carried out in the course of this work are described in $[3,4,6,7,8,9]$ and the papers resulting from this work are listed in the report, the remaining document will focus on the description of the spectrometer that was constructed.

In our experiments, we are able to observe energy conversion in state changing collisions by measuring the velocity distributions of the collision fragments. Figure 1 is an example of a crossing between two interatomic potentials that could give rise to a state changing collision. For a specific system, this could be an avoided crossing or a real crossing of two potential energy curves. By exciting the collision complex at the curve crossing, a pair of atoms will gain kinetic energy (KE) equal to the difference between the electronic energy at the curve crossing and the asymptotic electronic energy of the exit channel. By measuring the velocity determined by KE, the exit channel can be identified.

To measure the KE distributions resulting from ultracold collisions, we constructed a Rydberg tagging, photofragment time-of-flight spectrometcr. By exciting the collision products to high principal quantum number Rydberg states, the products are allowed to expand at the collision exit velocity as neutrals. Since the products expand as neutrals, they are less sensitive to stray electric fields that can destroy the fidelity of a measurement. Rydberg tagging is also a way to distinguish collision products from background gas atoms. 


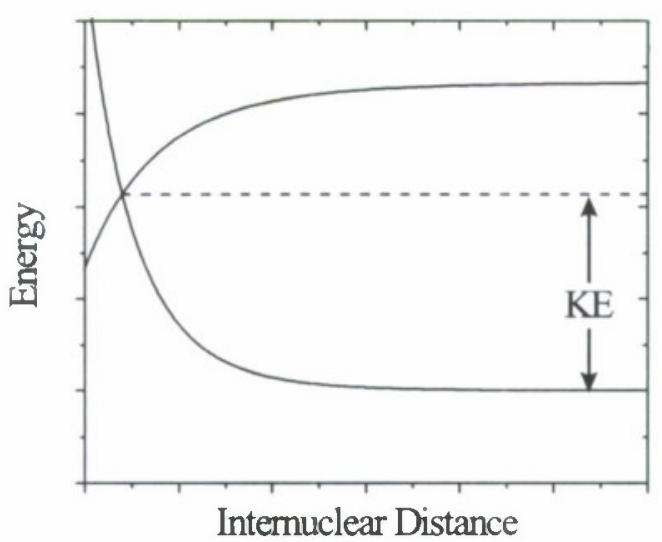

Figure 1: A pair of atoms excited at the curve crossing will gain velocity by the conversion of electronic energy to kinetic energy (KE). The collision pair will expand at a velocity determined by the gain in KE. The energy of the fragments can be used to determine the exit channels of the collision process.

Measurements pcrformed in our spectrometer begin in a magneto-optical trap (MOT). By intersecting the MOT with tightly focused probe beams $(<100 \mu \mathrm{m}$ diameter $)$, the excitation volume can be localized. It is important for the greatest energy resolution to start with the smallest excitation volume possible but maintain high product yield. Higher density can be achieved in a smaller volume using an optical dipole trap. Our apparatus has a dipole trap integrated into the setup, Figure 2.

The Rydbcrg tagged products are detected by pulsed-field ionization (PFI). By designing the spectrometer to apply a momentum kick to the products rather than using an acceleration region, the product distribution can be approximately linearly mapped onto a detector. Also, by appropriately shaping the PFI field, the need for grids can be eliminated. Grid transmission loss can substantially decrease the overall detcction probability of the system, which can be a problem with low yield collision studies.

The spectrometer is contained in a $22.9 \mathrm{~cm}$ radius stainless steel vacuum chamber (sce Figure 2). It is evacuated to a pressure of $2 \times 10^{-10}$ Torr by a turbo-molecular pump. The turbo-molecular pump is backed by a diffusion pump which is backed by a mechanical roughing pump. The backing of the turbo pump with a diffusion pump provides for a high compression ratio that makes it easier for the turbo pump to reach its base pressure.

A Cs MOT is formed in the center of the chamber. The experiments to date have relied on loading the MOT from a background vapor. The last modification that was done was to add a Zeeman slower to load the MOT so that larger atom numbers can be achieved. This will make our dipole trap easier to use for experiments. The MOT is formed using two home built diode lasers and a set of anti-Helmholtz coils. The anti-Helmholz coils for the MOT are located inside the chamber. The coils are square with a length of $14.7 \mathrm{~cm}$ on a side. The coils contain 24 windings each of Kapton coated, copper tubing. Water is circulated through the coils to dissipate heat. The coils are operated at $34 \mathrm{~A}$ for a magnetic field gradient of $12.5 \mathrm{G} \mathrm{cm}^{-1}$ at the MOT. 


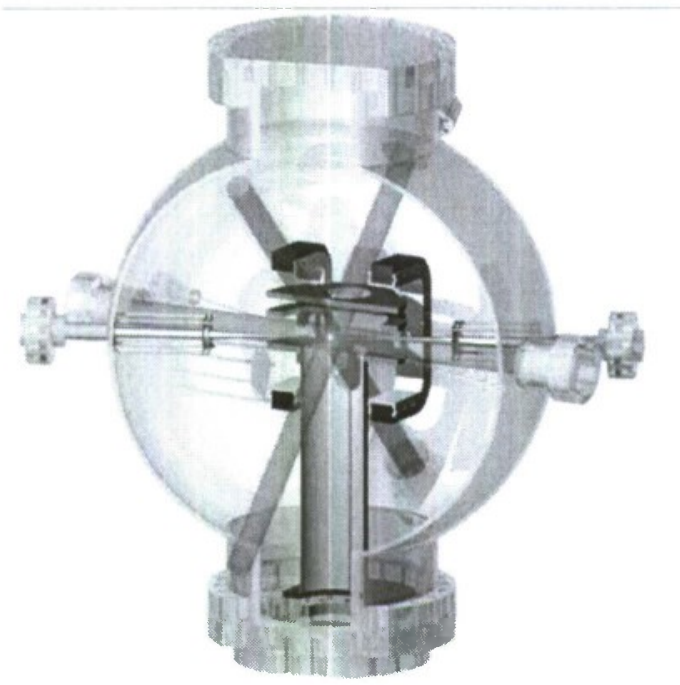

Figure 2: To scale rendering of the spectrometer located in the vacuum chamber. The spectrometer is sectioned for viewing. The bright spot in the center represents the MOT. The spectrometer is formed around the MOT with the flight tube extending downward towards the MCP detector. The anti-Helmholz coils are also depicted. The lens systems are used to focus a $\mathrm{CO}_{2}$ laser to form an optical dipole trap.

The spectrometer is centered on the MOT. Three stainless steel, circular field shaping plates are distributed above and below the MOT. $33 \mathrm{~mm}$ above the MOT is a plate with holes for two of the MOT trapping beams. The holes are filled with glass and covered with an electro formed $\mathrm{Ni}$ wire mesh 4.6 lines $\mathrm{mm}^{-1}$ on the side of the glass closest to the MOT. $19 \mathrm{~mm}$ below this plate is a field shaping ring. $28 \mathrm{~mm}$ below the shaping ring is a grounded plate with a $74 \mathrm{~mm}$ diameter hole. The MOT is located halfway between the bottom plate and the shaping ring. All three plates are separated by ceramic spacers. Connected to the bottom plate is a $25.4 \mathrm{~cm}$ grounded flight tube. The spectrometer is coated with Aerodag to minimize reflections and smooth out field imperfections. At the end of the flight tube is a $40 \mathrm{~mm} \mathrm{Z}$-stack cross delay-line (XDL) microchanncl plate (MCP) detector with a $19 \mu \mathrm{m}$ spatial resolution determined from spot size measurements of a pinhole mask illuminated with ultraviolet light.

The Rydberg atoms are projected onto the detector by applying a high voltage pulse to the top plate. The pulse ionizes the Rydberg atoms and transfers a momentum kick, $p=\int q E(t) d t$, to the ions in the MOT region. $q$ is the charge and $\mathrm{E}(\mathrm{t})$ the electric field pulse as a function of time. The pulse is applied using a HV pulser (DEI PVX-4140) powered by a high voltage supply (Glassman EK3R200). Resistors are symmetrically spaccd around the edges of the plates. The resistance between the top plate and shaping ring is $1 \mathrm{k} \Omega$ ( 4 resistors), and $2.1 \mathrm{k} \Omega$ between the shaping ring and bottom plate ( 4 resistors) for a combined resistance of $3.1 \mathrm{k} \Omega$. With an applied voltage of $320 \mathrm{~V}$, there is an electric field of $53 \mathrm{~V} \mathrm{~cm}^{-1}$ at the position of the MOT. The rise time of the pulse is $80 \mathrm{~ns}$ limited by the HV pulser. The fastest rise time achievable is $10 \mathrm{~ns}$, determined using a digital delay generator (SRS DG535). After the pulse, ions travel through the field free flight tube and are detccted on the MCP. 
Figure 3 shows the calculated electric equipotential lines in the region around the MOT, for an applied voltagc of $320 \mathrm{~V}$. The potential is calculated by numerically solving the Laplace-equation in a cylindrically symmetric $3 \mathrm{D}$ space using the program SIMION. The symmetry axis coincides with the center of the flight tube. The cross section of the electrode configuration is specified on a 2D grid of 400 horizontal points by 200 vertical points with a grid spacing of $1 \mathrm{~mm}$. The modeled electrodes match the spectrometer plate geometry. The top plate, the shaping ring, and the bottom plate and flight tube are modeled as ideal conductors at fixed potentials of $320 \mathrm{~V}$, $218 \mathrm{~V}$, and $0 \mathrm{~V}$, respectively. No other parts of the experimental setup are included in the simulation. Under these conditions, the longitudinal electric field at the MOT is calculated to be $E_{l}=52.2 \mathrm{~V} \mathrm{~cm}^{-1}$. The field gradient along the TOF axis of $8.87 \mathrm{~V} \mathrm{~cm}^{-2}$ at the MOT center implies that the field changes by $92 \mathrm{mV} \mathrm{cm}$ over a $100 \mu \mathrm{m}$ excitation volume in the time-of-flight direction. The radial electric field gradient at the MOT is $4.23 \mathrm{~V} \mathrm{~cm}^{-2}$. We assume no stray electric field for the simulation. From the calculated field at the MOT, we obtain a geometric factor $\alpha=\mathrm{E} / \mathrm{V}=0.163 \mathrm{~cm}^{-1}$ relating the applied voltage and the field at the MOT position.

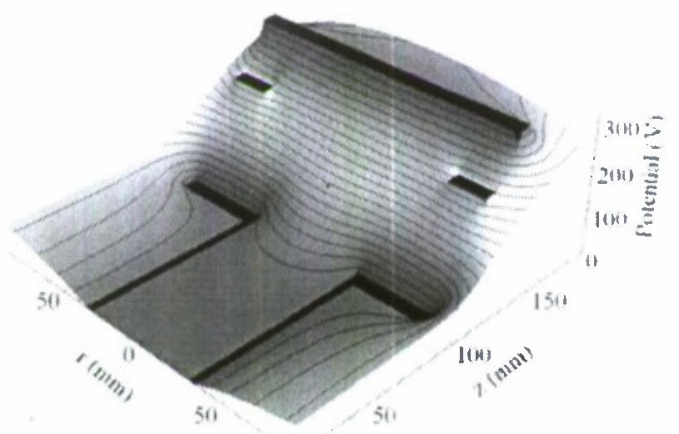

Figure 2: Plot of the equipotential surfaces with an applied voltage of $320 \mathrm{~V}$ on the top plate. The lensing of the field is due to the absence of a grid on the opening of the bottom plate. The MOT is displayed as a Gaussian distribution at its location on the surface.

Time-of-flight velocity distributions can be reconstructed using high speed electronics. The current monitor of the HV pulser is processed using a constant fraction discriminator (CFD) and serves as the start input for a 2 $\mathrm{GHz}$ multichannel analyzer (MCA) (Fast ComTec P7886). Fast timing signals (FWHM 2 ns) from the MCP anode are amplified using a preamplifier (SR240). The amplified signals are processed using a second CFD and serve as stop inputs for the MCA. The XDL grid beneath the MCP is designed for 2D imaging of product spatial distributions. The signals from the XDL grid are integrated by preamplifiers for $3 \mu$ s to providc a charge signal proportional to the number of ions incident on the detector. The stop signals can be filtered using the charge signals. The charge signals may be used to gate the fast timing signals by using a single channel analyzer to threshold the velocity distributions based on the height of the charge pulse. The chargc pulse height distribution (PHD) is also read by an analog to digital converter. The charge PHD is determined by the binomial distribution and is a measure of the overall detection probability of the system if the number of products in a collision is known. 
Our studies of the cold Rydberg gas have sought to untangle the complicated interactions between Rydberg atoms. We have recently begun to develop 3-dimensional velocity imaging, as our ultimate objective is to study three-body recombination at ultralow temperatures. These experiments will utilize the full three dimensional imaging capabilities of our apparatus. Three-body recombination is important in cold atom physics because it leads to loss from Bose-Einstein condensates. The problem of three-body recombination is intimately related to the coherent control of a bimolecular reaction, because three-body break-up is a possible exit channel in any experiment attempting to coherently control a bimolecular reaction. Our studies of 3-body recombination promise to be the first multiply differential studies of a chemical process where a bond is formed and broken. The apparatus described here is then important for the development of the field of chemistry at ultracold temperatures.

[1] "Sub-Doppler Magneto-Optic-Trap Temperatures Measured Using Rydberg Tagging," J. Tallant, K.R. Overstreet, A. Schwettmann, and J.P. Shaffer, Physical Review A, 74, 023410/1 (2006).

[2] "Photoinitiated Collisions Between cold Cs Rydberg Atoms," K.R. Overstreet, J. Tallant, A. Schwettmann, and J.P. Shaffer, Physical Review A, Rapid Communications, 76, 1 1403(R)/1 (2007).

[3] “Cold Cs Rydberg Gas Interactions," A. Schwettmann, J. Crawford, K.R. Overstreet, and J.P. Shaffer, Physical Review A, Rapid Communications, 74, $020701(\mathrm{R}) / \mathrm{I}(2006)$.

[4] "Long Range Cs Rydberg Molecules," A. Schwettmann, K.R. Overstreet, and J.P. Shaffer, Journal of Modern Optics, 54, 2551 (2007).

[5] "Observation of Cs Rydberg atom macrodimers," K.R. Overstreet, J. Tallant, A. Schwettmann, D. Booth and J.P. Shaffer, Nature Physics, submitted for publication (arXiv:0901.1825v1).

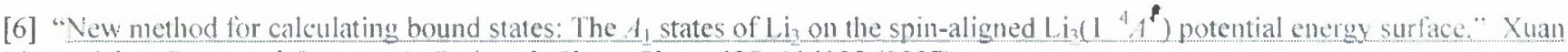
Li, Daniel A. Brue, and Gregory A. Parker, J. Chem. Phys., 127, 014108 (2007).

[7] "Potential energy surfaces for the 14A', $24 \mathrm{~A}$ ' $14 \mathrm{~A}$ " and $24 \mathrm{~A}$ " states of Li3,"

Xuan Li, Daniel A. Brue, and Gregory A. Parker, J. Chem. Phys., 129, 124305 (2008) .

[8] "Theory of laser enlancement of ultracold reactions: The fermion-boson population transfer by adiabatic passage of ${ }^{6} \mathrm{Li}+{ }^{6} \mathrm{Li}{ }^{7} \mathrm{Li}\left(T_{r}=1 \mathrm{mK}\right) \rightarrow{ }^{6} \mathrm{Li}{ }^{6} \mathrm{Li}+{ }^{7} \mathrm{Li}\left(T_{p}=1 \mathrm{mK}\right), "$ Xuan Li and Gregory A. Parker, J. Chem. Pliys., 128, 184113 (2008).

[9] "Laser-Catalyzed Production of Ultracold Molecules: The ${ }^{6} \mathrm{Li}+{ }^{6} \mathrm{Li}{ }^{7} \mathrm{Li} \rightarrow h \omega+{ }^{6} \mathrm{Li}-{ }^{6} \mathrm{Li}+{ }^{7} \mathrm{Li}$ Reaction ," Xuan Li, Gregory A. Parker, Paul Brumer, loannis Thanopulos, and Moshe Shapiro, Phys. Rev. Lett., 101, 043003 (2008). 


\section{Papers Published, in Sumission, or Preparation}

1. "Multiple Scattering and the Density Distribution of a Cs MOT," R. Overstreet, P. Zabawa, J. Tallant, A. Schwettmann, and J. Shaffer, Optics Express, 24, 9672 (2005).

2. "Cold Cs Rydberg Gas Interactions," A. Schwettmann, J. Crawford, K.R. Overstreet, and J.P. Shaffer, Pliysical Rcview A, Rapid Communications, 74, $020701(\mathrm{R}) / 1$ (2006).

3. "Sub-Doppler Magneto-Optic-Trap Temperatures Measured Using Rydberg Tagging," J. Tallant, K.R. Overstreet, A. Schwettmann, and J.P. Shaffer, Physical Review A, 74, 023410/1 (2006).

4. "A Tunable Four Pass Narrow Spectral Bandwidth Amplifier for Use at $\sim 500 \mathrm{~nm}, "$ A. Schwettmann, C. McGuffy, S. Cliaulan, K.R. Overstreet and J.P. Shaffer, Applied Optics, 46, 1310 (2007).

5. “Photoinitiated Collisions Between cold Cs Rydberg Atoms," K.R. Overstreet, J. Tallant, A. Schwettnann, and I.P. Shaffer, Phys. Rev. A, Rapid Communications, 76, $11403(\mathrm{R}) / 1$ (2007).

6. “Long Range Cs Rydberg Molecules," A. Schwettmann, K.R. Overstreet, and J.P. Shaffer, Journal of Modern Optics, 54, 2551 (2007).

7. "Laser-Catalyzed Production of Ultracold Molecules: The ${ }^{6} \mathrm{Li}+{ }^{6} \mathrm{Li} \mathrm{Li} \rightarrow \hbar \omega+{ }^{6} \mathrm{Li}-{ }^{6} \mathrm{Li}+{ }^{7} \mathrm{Li}$ Reaction, " Xuan Li, Gregory A. Parker, Paul Brumer, loannis Thanopulos, and Moshe Shapiro, Phys. Rev. Lett., 101, 043003 (2008).

8. "Potential cnergy surfaces for the $1^{4} A^{\circ}, 2^{4} A^{\prime} 1^{4} A$ " and $2^{4} A$ " states of $\mathrm{Li}_{3}$," Xuan Li, Daniel A. Brue, and Gregory A. Parker, J. Chem. Phys., 129, 124305 (2008).

9. "Theory of laser enhancemcnt of ultracold reactions: The fermion-boson population transfer by adiabatic passage of ${ }^{6} \mathrm{Li}+{ }^{6} \mathrm{Li}{ }^{7} \mathrm{Li}\left(T_{r}=1 \mathrm{mk}\right) \rightarrow{ }^{6} \mathrm{Li}{ }^{6} \mathrm{Li}+{ }^{7} \mathrm{Li}\left(T_{p}=1 \mathrm{mK}\right), "$ Xuan Li and Gregory A. Parker, J. Chem. Phys., 128, 184113 (2008).

10. "New method for calculating bound states: The $A_{1}$ states of $\mathrm{Li}_{3}$ on the spin-aligned $\mathrm{Li}_{3}\left(\mathrm{l}^{4} A^{\prime}\right)$ potential energy surface," Xuan Li, Daniel A. Brue, and Gregory A. Parker, J. Chem. Phys., 127, 014108 (2007).

11. "Observation of Cs Rydberg atom macrodimers," K.R. Overstreet, J. Tallant, A. Schwettmann, D. Booth and J.P. Shaffer, Nature Physics, submitted for publication (arגiv:0901.1825v1).

12. "Photofragment Spectrometer for Ultracold Collisions," K. R. Overstreet, A. Schwettmann, J. Tallant, C. Austin and J.P. Shaffer, Applied Physics B, in preparation.

13. "Controlled formation of ultracold NaCs molecules by photoassociation via adiabatic passage," Xuan Li, William Dupre, Michael A. Morrison, James P. Shaffer and Gregory A. Parker, Submitted to Physical Review A for publication.

\section{$\underline{\text { Talks and Conference Presentations }}$}

1. "Cold Cs. Rydberg Atom Collisions," J. Shaffer, ITAMP - Workshop on Ultracold Rydbcrg Gases and Plasmas, ITAMP, (2005). (invited)

2. "Excitations in an Ultracold Rydberg Gas," J. Shaffer, International Workshop on Correlated and Many-Body Phenomena in Dipolar Systems, Max Planck Institute for Complex Systems, Dresden Germany (2006). (invited)

3. "Rydberg Atom - Rydberg Atom Dipole - Dipole Potentials," A. Schwettmann, J. Crawford, K. R. Overstreet, and J.P. Shaffer, DAMOP, Knoxville, TN, (2006).

4. "Temperature Measurements Using Rydberg Tagging," J. Tallant, K.R. Overstreet, A. Schwettmann, J. Crawford, and J.P. Shaffer, DAMOP, Knoxville, TN, (2006).

5. "Abel Inversion for Study of Multiple Scattering in a Cs Magneto-Optic Trap," K.R. Overstreet, P. Zabawa. J. Tallant, A. Schwettmann, J. Crawford, and J.P. Shaffer, DAMOP, Knoxville, TN, (2006). 
6. "Ultracold Cs Rydberg Gas Dynamics," K.R. Overstreet, A. Sehwettmann, J. Tallant and J.P. Shaffer, OSA/LS, Rochester, NY, (2006).

7. "Long Range Cs Rydberg Molecules," A. Schwettmann, K.R. Overstreet, J. Tallant and J.P. Shaffer, DAMOP. Calgary, Canada, (2007).

8. "Rydberg Tagging Time-of-Flight lmaging to Study Ultracold Collisions," J. Tallant, K.R. Overstreet, A. Schwettmann and J.P. Shaffer, DAMOP, Calgary, Canada, (2007).

9. "Resonant Collision Processes in a Cs Rydberg Gas," K.R. Overstreet, A. Schwettmann, J. Tallant, and J.P. Shaffer, DAMOP, Calgary, Canada, (2007).

10. "Dynamies of an Ultracold Cs Rydberg Gas," J.P. Shaffer, Physies of Quantum Electronies, Snowbird, UT, (2007). (invited)

11. "Long Range Cs Rydberg Molecules," A. Schwettmann, K.R. Overstreet, J. Tallant, and J.P. Shaffer, OSA/LS, Long Beach, CA (2007).

12. “Dipole-Dipole Interactions in a Cold Cs Rydberg Gas," J. Tallant, K.R. Overstreet, A. Schwettmann and J.P. Shaffer, DAMOP, State College (2008).

13. "Long Range, Cold Cs Rydberg Atom-Rydberg Atom Molecules," K.R. Overstreet, A. Sehwettmann, J. Tallant, and J.P. Shaffer, DAMOP, State College (2008).

14. "Long Range, Cold Cs Rydberg Atom-Rydberg Atom Molecules," J.P. Shaffer, Common Perspectives of Mesoscopic Systems and Quantum Gases, Gunzberg, Germany (2008).

15. "Cold Cs Rydberg Molecules," J.P. Shaffer, Lundbeck Theory Center Workshop on Rydberg Atom Physies at Sandbjerg Estate, Denmark. (invited)

16. "Cold Atoms and Inertial Navigation," J.P. Shaffer, The Tulsa Astronomy Society, Tulsa, OK (2006). (iwvited)

17. "Cold Cs Rydberg Atom Collisions," J.P. Shaffer, UNC Charlotte, (2006). (invited)

18. "Cold Rydberg Gas Interactions," J.P. Shaffer, University of Oklahoma, (2007). (invited)

19. "Dynamies of an Ultracold Cs Rydberg Gas," J.P. Shaffer, University of Arkansas, (2007). (iuvited)

20. “Cold Cs Rydberg Gas Interactions," J.P. Shaffer, University of Stuttgart, Stuttgart, Germany (2008). (invited)

21. "Cold Cs Rydberg Gas Interactions," J.P. Shaffer, University of Tubingen/University of Stuttgart/University of Ulm SSB colloquim. (invited)

22. "Controlled formation of diatomic molecules using laser catalysis," X. Li and G. Parker, DAMOP, State College (2008).

23. "Controlled formation of diatomie molecule using laser catalysis," X. Li and G. Parker, DAMOP, State College (2008).

24. "Controlled formation of diatomic molecule using laser catalysis," X. Li and G. Parker, ICAP, Storrs (2008).

\section{Theses Resulting from this Work}

K. Richard Overstreet, "Experimental Studies of Cesium Rydberg Atom Pair Interactions," The University of Oklahoma (2008).

Xuan Li, "Coherent Control and Geometric Phase," The University of Oklahoma (2008). 


\begin{abstract}
$\underline{\text { Abstract }}$
In this work, a Rydberg atom imaging spectrometer to study individual collision events in an ultracold gas was developed. The apparatus achieved velocity resolution of $2.5 \mathrm{~cm} / \mathrm{s}$. This resolution allows all known ultracold collision processes to be resolved. The spectrometer was tested on low energy Rydberg atom collisions occurring in an ultracold gas of Cs atoms. To analyze this data, a procedure for calculating the interactions between high lying Rydberg states was developed. A parallel computer code to calculate these interactions was completed and implemented on a cluster machine (OSCER). The potentials were used to predict the results of the Rydberg atom collisions studied with the spectrometer. The theory and experiments were found to be in excellent agreement. The Rydberg interaction potentials are important for understanding and predicting the characteristics of dipole blockade which is important for quantum computation schemes that use atoms. Calculations were also completed on the Li trimer to describe 3-body recombination and other ways that molecules can form in ultracold gases. The goal of these calculations was to predict differential cross-sections for 3 -body recombination so that future measurements with the imaging spectrometer that was built and tested on Rydberg collisions can be compared to this theory.
\end{abstract}

\title{
Outreach
}

Shaffer gave a talk at the meeting of the Tulsa Astronomy club.

6 undergraduates participated in this project either through the NSF-REU program or through their senior thesis project.

\section{Consultive and Advisorv Functions}

Richard Overstreet visited the atom chip group at Hanscom Air Force Base to help with experiments there on 2 different occasions. (Jan. 22 - Feb. 8, 2008) (July 20-Aug. 4, 2007)

Undergraduate Erin Brown an Air Force ROTC officer did her undergraduate thesis in our research group. Sher was assigned to do research in the Air Force Research Labs (Spring 2008 - Spring 2009).

\section{Honors}

James Shaffer - Fellow of the Alexander von Humboldt Society

Gregory Parker - George Lynn Cross Research Professor

\section{Personnel Supported}

James Shaffer (PI)

Greg Parker (co-PI)

Jonathan Tallant (Ph.D. Student)

Arne Schwettman (Ph.D. Student)

K. Richard Overstreet (Ph.D. Student)

J. Crawford (Ph.D. Student) 